\title{
Enhancing safe prescribing using a theory-informed feedback intervention
}

\author{
Ian Coombes ${ }^{1}$, Peter Donovan ${ }^{2}$, Charles Mitchell ${ }^{3}$, Brooke Bullock ${ }^{4}$, and Christy Noble ${ }^{5}$ \\ ${ }^{1}$ Royal Brisbane \& Women's Hospital \\ ${ }^{2}$ Royal Brisbane and Women's Hospital \\ ${ }^{3}$ University of Queensland \\ ${ }^{4}$ Gold Coast University Hospital \\ ${ }^{5}$ The University of Queensland Faculty of Medicine and Biomedical Sciences
}

February 3, 2022

\begin{abstract}
Context Medical interns find prescribing challenging and lack readiness when commencing work. Error rates remain high despite education, supervision and pharmacists' contributions. Feedback on prescribing may improve performance. Yet, work-based prescribing feedback focuses on rectifying errors. We aimed to improve prescribing using a theory-informed feedback intervention. Methods In this mixed methods study, we designed and implemented a feedback intervention, informed by Feedback-Mark 2 Theory. Interns commencing internal medicine terms engaged in the intervention. Prescribing performance was evaluated by comparing the number of errors per order of at least 30 medication orders per prescriber from weeks 1-3 (pre/baseline) and weeks 8-9 (post-intervention). Baseline interns' audit findings were analysed and discussed during individualised feedback sessions with either a clinical pharmacologist (Site 1) or pharmacist educator (Site 2). Results Prescribing by 88 interns, across two hospitals over five 10 week terms, was analysed. Following the intervention, there was a significant reduction in prescribing errors $(\mathrm{p}<0.001)$ from 1598 errors in 2750 initial orders (median [IQR] 0.48 [0.35-0.67] errors per order) before and 1113 errors in 2694 orders (median [IQR] 0.30 [0.17-0.50] errors per order) after. There was no reduction in initial error rate over the five terms. Conclusions Our theory-informed feedback intervention contributed to a significant reduction in interns' prescribing errors. This study opens up new strategies for improving prescribing safety through feedback interventions. The results emphasise that learner centred and improvement-focussed feedback with an agreed plan could significantly improve prescribing safety an outcome which supervision, education and pharmacist interventions had not delivered.
\end{abstract}

\section{Hosted file}

Intern prescriber FBK_vUpload.docx available at https://authorea.com/users/458732/articles/ 555208-enhancing-safe-prescribing-using-a-theory-informed-feedback-intervention

\section{Hosted file}

Fig1_error per order per precriber .docx available at https://authorea.com/users/458732/ articles/555208-enhancing-safe-prescribing-using-a-theory-informed-feedback-intervention

\section{Hosted file}

table 1 timeline and overview of study .docx available at https://authorea.com/users/458732/ articles/555208-enhancing-safe-prescribing-using-a-theory-informed-feedback-intervention

\section{Hosted file}


Table 2_Demographic data .docx available at https://authorea.com/users/458732/articles/ 555208-enhancing-safe-prescribing-using-a-theory-informed-feedback-intervention 